\title{
Circular dichroism of four-wave mixing in nonlinear metamaterials
}

\author{
Alec Rose, ${ }^{1}$ David A. Powell, ${ }^{2}$ Ilya V. Shadrivov, ${ }^{2}$ David R. Smith, ${ }^{1}$ and Yuri S. Kivshar ${ }^{2,3}$ \\ ${ }^{1}$ Center for Metamaterials and Integrated Plasmonics, Duke University, Durham, North Carolina 27708, USA \\ ${ }^{2}$ Nonlinear Physics Center, Research School of Physics and Engineering, Australian National University, \\ Canberra, Australian Capital Territory 0200, Australia \\ ${ }^{3}$ National Research University of Information Technologies, Mechanics and Optics, St. Petersburg 197101, Russia \\ (Received 6 September 2013; revised manuscript received 11 November 2013; published 25 November 2013)
}

\begin{abstract}
Metamaterial engineering offers a route to combine unusual and interesting optical phenomena in ways that are rare or nonexistent in nature. As an exploration of this wide parameter space, we experimentally demonstrate strong cross-phase modulation and four-wave mixing in a chiral metamaterial, highlighting the interplay of nonlinearity and circular dichroism. Furthermore, we show that the magnitude of the nonlinear parametric interaction follows certain selection rules regarding the circular polarizations of the various interacting waves. Using a coupled-mode analysis and finite element simulations, we relate these selection rules to the metamaterial's internal symmetries as well as its circular dichroism in the linear regime.
\end{abstract}

DOI: 10.1103/PhysRevB.88.195148

PACS number(s): 81.05.Xj, 42.70.Mp, 78.20.Ek

\section{INTRODUCTION}

The metamaterial concept represents a powerful tool for designing electromagnetic components with multiplex functionalities based on frequency, direction, and polarization. Recently, chiral metamaterials have become a particularly active area of research, with demonstrations of giant optical activity and circular dichroism. ${ }^{1-6}$ In most of those studies, the chiral metamaterials are planar in nature, implementing chirality through a rotation in the orientation of metallic elements in progressive planes. In such an arrangement, the resonances of the individual elements interact to give preference to one particular circular or elliptic plane-wave polarization. This results in strongly polarization-dependent optical properties, supporting massive rotatory power and/or circular dichroism in geometrically tunable structures.

When combined with nonlinear components, the degrees of freedom in metamaterial engineering grow further, including a number of important nonlinear effects such as self- and crossphase modulation, frequency conversion, and even parametric amplification. ${ }^{7-10}$ In many cases, the intrinsic resonance of the metamaterial is coupled directly to a nonlinear component or material, imbuing the metamaterial with nonlinear properties that can be many times enhanced compared to its constituents alone. Varactor-loaded metamaterials, for example, have provided convenient proof-of-concept microwave demonstrations of various enhanced or novel nonlinear processes in metamaterials, such as wave mixing, ${ }^{11,12}$ resonance tuning, ${ }^{8,13,14}$ and negative-index second-harmonic generation. ${ }^{15}$

Since a lack of inversion symmetry is a primary feature of both chiral and second-order nonlinear materials, these phenomena have been studied together in a number of materials with both microscopic and macroscopic chiral ordering. ${ }^{16-18}$ In particular, nonlinear chiral metamaterials have been implemented as a first step toward power-dependent polarization rotators. ${ }^{19-21}$ However, such demonstrations only begin to probe the parameter space for simultaneous chirality and nonlinearity.

In this paper, we study the interplay of third-order nonlinearity and chirality in nonlinear metamaterials, focusing on the role of circular dichroism in both cross-phase modulation and parametric four-wave mixing. We characterize the nonlinear response for waves of different circular polarization, relating the relative magnitudes to the metamaterial's linear circular dichroism and symmetries. For the example of a varactorloaded metamaterial, we demonstrate experimentally a type of nonlinear chiral metamaterial which exhibits strong four-wave mixing, in agreement with the derived selection rules.

The paper is organized as follows. In Sec. II we discuss the nonlinear third-order response of metamaterials and introduce the nonlinear four-wave interaction and corresponding coefficients. We also introduce the metamaterial's circular dichroism in its third-order nonlinear response. Section III discusses our design of a nonlinear metamaterial with varactor diodes. In Sec. IV we present our experimental and numerical results on two types of third-order nonlinear effects, namely, the cross-phase modulation and four-wave mixing. Finally, Sec. V concludes the paper.

\section{NONLINEAR WAVE MIXING IN METAMATERIALS}

In stark contrast to natural materials, the nonlinear interaction between electric and magnetic fields of varying polarizations can be specifically tailored in a metamaterial. While this greatly enhances the degrees of freedom available in typical parametric and modulation processes, it also implies greater complexity. Several methods and formalisms have been proposed to frame these interactions in the typical language of nonlinear optics by using effective nonlinearities, such as transfer-matrix based retrieval methods. ${ }^{22-24}$ Alternatively, a coupled-mode theory was developed in order to explore the mixing of both magnetic and electric fields, exploiting Bloch modes in the context of periodic metamaterials. ${ }^{25}$ This approach has the advantage of yielding quasianalytic expressions for the effective nonlinear coupling coefficients, providing intuition regarding symmetries and unit-cell design. Thus, for a uniaxial chiral metamaterial in which waves propagating along the optical axis can be decomposed into right circularly polarized $(\mathrm{RCP},+)$ and left circularly polarized (LCP, -) waves, coupled-mode theory can reveal selection rules regarding the parametric interaction of multiple waves with distinct polarizations. 
As an example, we consider the four-wave mixing between pump $\left(\omega_{p}\right)$ and signal $\left(\omega_{s}\right)$ waves to parametrically generate a wave at frequency $\omega_{n l}=2 \omega_{p}-\omega_{s}$. Following conventional coupled-mode theory, ${ }^{26,27}$ we can assume coupling equations of the form

$$
\frac{\partial A_{n l}^{i}}{\partial z}=i \Gamma_{i j j k}\left(A_{p}^{j}\right)^{2}\left(A_{s}^{k}\right)^{*} e^{i \Delta k z},
$$

where indices $i, j, k \in[+,-]$ denote the waves' circular polarizations, each wave's intensity is given by $2|A|^{2}$, and $\Delta k=2 k_{p}^{j}-k_{s}^{k}-k_{n l}^{i}$. The key parameter in this equation is the coupling coefficient, $\Gamma_{i j j k}$, which we expect to take the form of an overlap integral. The ordering of the subscripts of the coupling coefficient follows the standard convention, in which the first subscript refers to the generated wave (with frequency $\omega_{n l}$ in our case) and the next three subscripts denote the fundamental driving waves. The efficiency of the wave-mixing process, which we define as $\eta=I_{n l} / I_{p}^{2} I_{s}$, is thus proportional to the square modulus of the coupling coefficient.

Meanwhile, we can fully describe the local properties of the metamaterial through a periodic relative permittivity, $\epsilon(\vec{r})$, and second-order electric nonlinearity, $\chi_{\mathrm{loc}}^{(2)}(\vec{r})$, such that $\epsilon(\vec{r})=\epsilon(\vec{r}+\vec{R})$ for any lattice vector $\vec{R}$, and likewise for the nonlinearity. Thus, in the linear regime, the electric and magnetic fields can be decomposed into Bloch modes ${ }^{28}$ such that Bloch mode $\mu$ is given by

$$
\begin{aligned}
\vec{E}_{\mu}(\vec{r}) & =A_{\mu} \vec{e}_{\mu}(\vec{r}) \exp \left(i \vec{k}_{\mu} \cdot \vec{r}\right), \\
\vec{H}_{\mu}(\vec{r}) & =A_{\mu} \vec{h}_{\mu}(\vec{r}) \exp \left(i \vec{k}_{\mu} \cdot \vec{r}\right),
\end{aligned}
$$

where $A_{\mu}$ is the mode amplitude, $k_{\mu}$ is the Bloch wave vector, and $\vec{e}_{\mu}(\vec{r})$ and $\vec{h}_{\mu}(\vec{r})$ are periodic electric and magnetic Bloch functions, respectively.

By applying a volume integral to the usual expression of Lorentz reciprocity in the presence of a perturbation, in this case the local second-order nonlinearity, we can describe the above example of four-wave mixing in a periodic, uniaxial metamaterial according to ${ }^{25}$

$$
\begin{aligned}
& \frac{1}{2} \iiint_{V_{0}} \frac{\partial}{\partial z} A_{n l}^{i} d V \\
& =i \omega_{n l}\left(A_{p}^{j}\right)^{2}\left(A_{s}^{k}\right)^{*} \iiint_{V_{0}} \overline{\bar{\chi}}_{\mathrm{loc}}^{(2)}(\vec{r}): \vec{e}_{p}^{j}(\vec{r}) \vec{e}_{p}^{j}(\vec{r}) \vec{e}_{s}^{k}(\vec{r})^{*} \\
& \quad \cdot \vec{e}_{n l}(\vec{r})^{*} e^{i \Delta k z} d V,
\end{aligned}
$$

where the integral is taken over a single unit cell with volume $V_{0}$. In the limit of a sufficiently small unit cell, $(|\Delta k a| \ll 1)$, a comparison of this expression with Eq. (1) implies that the coupling coefficient in our metamaterial is given by

$$
\Gamma_{i j j k}=\frac{\omega_{n l}}{V_{0}} \iiint_{V_{0}} d V \overline{\bar{\chi}}_{\mathrm{loc}}^{(3)}(\vec{r}): \vec{e}_{p}^{j}(\vec{r}) \vec{e}_{p}^{j}(\vec{r})\left[\vec{e}_{s}^{k}(\vec{r})\right]^{*} \cdot\left[\vec{e}_{n l}^{i}(\vec{r})\right]^{*} .
$$

Thus, using Eq. (4), we can use the metamaterial symmetry and properties to derive a set of selection rules. For example, for a cubic unit cell, the uniaxial nature implies invariance for a $90^{\circ}$ rotation about the $z$ axis, denoted $\mathcal{R}_{\pi / 2}$, in the local material properties. Using this symmetry and the fact that the macroscopic eigenmodes are circularly polarized, we can infer

$$
\vec{e}_{n}^{ \pm}(\vec{r})= \pm \mathcal{R}_{\pi / 2}\left[\vec{e}_{n}^{ \pm}\left(\mathcal{R}_{-\pi / 2}[\vec{r}]\right)\right]
$$

From these symmetries, we see that certain combinations of circularly polarized waves are forbidden from interacting, since the four-wave mixing coefficient vanishes identically. In particular, the degeneracy of the pump wave implies that the only nonzero coupling coefficients are those involving signal and mix waves with the same circular polarization, or

$$
\eta_{i j j k}=0, \quad \text { for } i \neq k \text {. }
$$

Additionally, it is often the case that only one polarization can strongly excite an intrinsic resonance in the metamaterial, which can lead to giant circular dichroism. ${ }^{5}$ This implies enhancement of parametric coupling for the polarization which strongly excites the resonance, with greater enhancement expected for coefficients involving more terms with this polarization.

However, many of the previous assumptions are not always true for chiral metamaterials, and, furthermore, fabrication errors and finite-sized lattices will break their strict application. Nevertheless, one can infer certain qualitative features from the previous discussion. For example, if we consider a metamaterial supporting a resonance predominantly excited by RCP waves, and assume that all incident waves share the same polarization, it follows that

$$
\begin{gathered}
\eta_{++++} \gg \eta_{-+++}, \\
\eta_{----} \gg \eta_{+---}, \\
\eta_{++++} \gg \eta_{----} .
\end{gathered}
$$

The validity of the first two inequalities depends on the level to which the symmetry and periodicity assumptions are violated, while the third inequality depends on the metamaterial's circular dichroism. We note that although this coupled-mode theory was developed for bulk metamaterials, nonlinear interactions in thin samples are qualitatively similar, with the exception that the effect of phase mismatch is negligible. As with the linear properties of metamaterials, it cannot be assumed that the nonlinear coefficients of a bulk metamaterial have the same value as those of a single layer.

\section{STRUCTURE DESIGN AND CHARACTERIZATION}

To verify the previous discussion and, particularly, Eqs. (7)(9), we employ the varactor-loaded chiral metamaterial shown in Fig. 1. The structure resembles the canonical spiral, ${ }^{29,30}$ flattened such that it can be fabricated by a combination of standard photolithographic methods and vias. The fundamental resonance of these meta-atoms has strongly coupled parallel magnetic and electric dipole moments, leading to a strong chiral response. Moreover, nearest neighbors in the transverse plane are $90^{\circ}$-rotated replicas of the central element for the sake of isotropy, such that we can expect the transmission eigenmodes of the metamaterial to be circularly polarized. Nonlinearity is introduced by inserting a variable capacitance diode, or varactor, into each element. ${ }^{8,14}$ The unit cell was designed through a combination of finite-element simulations (COMSOL multiphysics) and standard scattering-based retrieval 


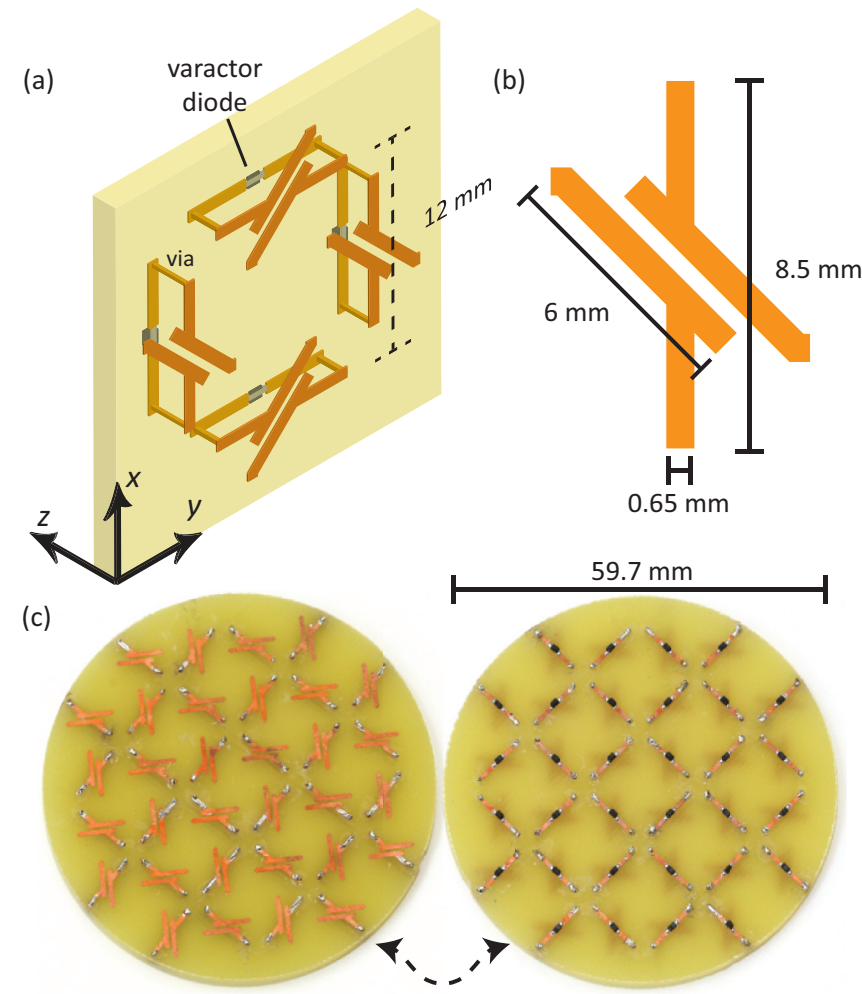

FIG. 1. (Color online) (a) Metamaterial unit cells, each consisting of two nonlinear particles. (b) Details of one side of the nonlinear particle. (c) and (d) Photographs of the two sides of the fabricated sample.

techniques, ${ }^{31}$ modeling the linear properties of the varactor diodes as lumped capacitive and resistive elements.

We compute the normal-incidence scattering parameters for a single unit cell with periodic boundary conditions, calculating the effective material properties by assuming a lattice spacing of $a=12 \mathrm{~mm}$ in the direction of propagation. This spacing is chosen to satisfy the usual metamaterial requirement of subwavelength unit cells $(a \approx \lambda / 7)$, while also minimizing the potential interaction between successive planes. The length and angle of the capacitive arms are optimized to provide maximum circular dichroism, which can be seen in the different complex indices of refraction for the two modes in Fig. 3(a). To illustrate how the two circularly polarized waves excite the system, in Fig. 2 we plot the modes of the structure excited by RCP and LCP waves at resonance. Similar circulating currents can be seen in each case, but with a difference in magnitude due to optimal coupling when excited by RCP waves.

The planar copper patterns were fabricated by photolithography on a 1.6-mm-thick FR4 substrate, while the vias and varactor diodes were subsequently soldered into place. We characterize the sample, shown in Fig. 1(c), in a circular waveguide with a 59.7-mm diameter. Using linear-to-circular polarization converters, we launch and collect signals for all permutations of right and left circular polarizations with a Rohde and Schwarz ZVB20 vector network analyzer (VNA). To lessen the effect of imperfect impedance matching at the circular waveguide ports, we measure transmission coefficients for six distinct positions of the sample within the waveguide, effectively sampling and averaging out the standing-wave pattern of the empty waveguide. The transmission coefficients for RCP and LCP modes are plotted in Fig. 3(b), while the polarization conversion is found to be suppressed by at least $10 \mathrm{~dB}$, indicating that the sample's eigenmodes are nearly circularly polarized. Moreover, it is clear that the system supports significant circular dichroism, reaching a maximum of $\sim 7 \mathrm{~dB}$ at the metamaterial resonance. For comparison, we also show the transmission coefficients from full-wave simulations of a semi-infinite slab of the metamaterial, showing agreement in the pertinent features.

\section{NONLINEAR EFFECTS}

Our experimental arrangement for nonlinear measurements is shown in Fig. 4. An HP 8673B signal generator provides a pump signal, which is amplified by an HP 83020A microwave amplifier, with a maximum output of $\sim 32 \mathrm{dBm}$. The VNA is used as the source of a low-amplitude probe signal and is combined with the pump using a 3-dB combiner before entering the coaxial-waveguide converter. In this configuration, we are able to investigate the parametric mixing of waves within the operational band of the waveguide and polarization elements, making cross-phase modulation and nearly degenerate four-wave mixing natural choices. We study the regime where the pump and signal have the same polarization.
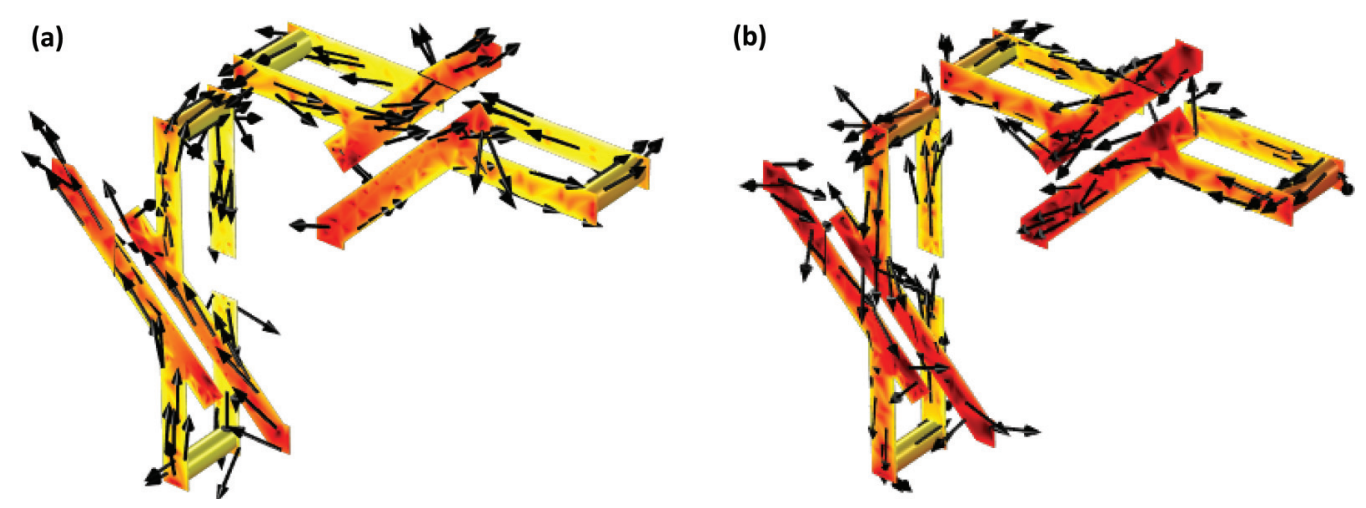

FIG. 2. (Color online) Modes of the structure excited by (a) right-handed and (b) left-handed circularly polarized waves. Arrows represent the direction of the surface currents while the magnitude is given in false color. 


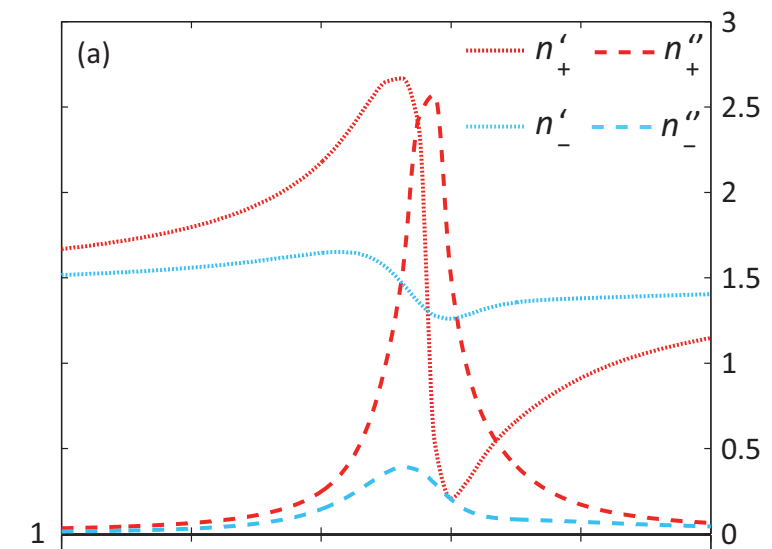

(b)

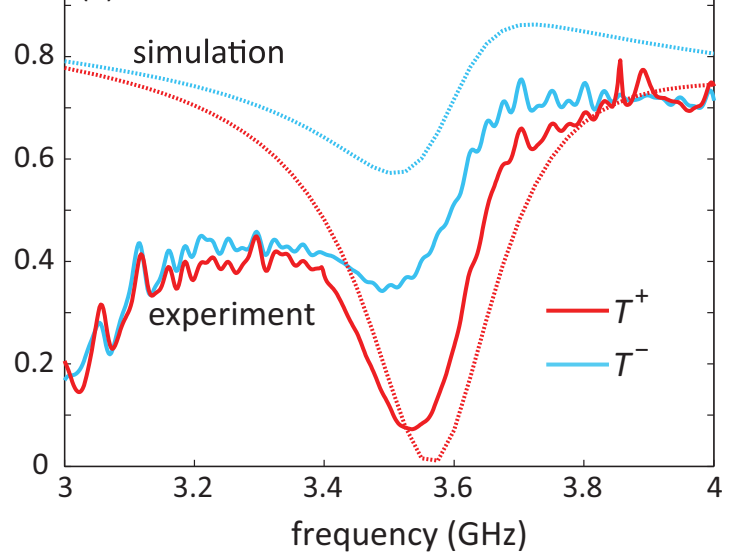

FIG. 3. (Color online) (a) Simulated refractive indices of the varactor loaded spirals, optimized to exhibit strong circular dichroism around $3.54 \mathrm{GHz}$. (b) Comparison of simulated and experimental transmission coefficients for both circular polarizations.

\section{A. Cross-phase modulation}

We first report cross-phase modulation of a weak signal wave by a strong pump wave, which is the most readily observable form of nonlinearity in this system. As has been demonstrated previously in varactor loaded metamaterials, ${ }^{14,20}$ sufficient incident pump powers can induce a change in the varactor's capacitance, leading to resonance tuning, in turn changing the transmission properties seen by a weak probe wave. Since the sample's resonance is directly responsible for
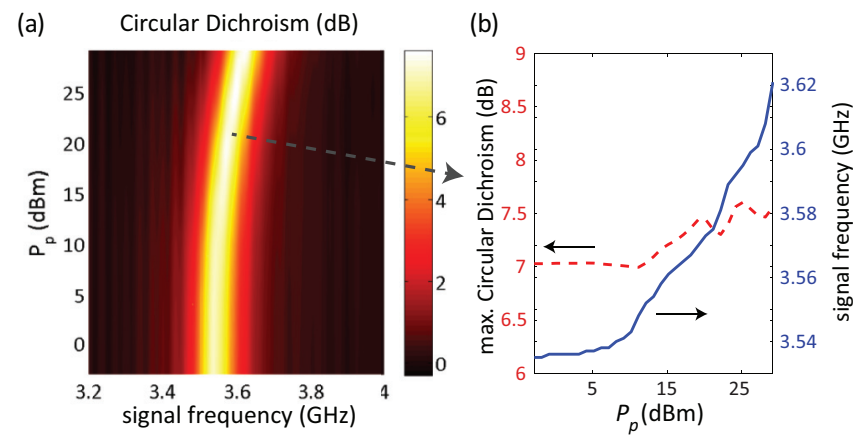

FIG. 5. (Color online) Experimental results for the cross-phase modulation effect. (a) Difference in transmission spectra for RCP and LCP waves as a function of the power of an additional pump wave, $P_{p}$, with $\omega_{p}=2 \pi \times 3.536 \mathrm{GHz}$. (b) Maximum circular dichroism magnitude and frequency as a function of $P_{p}$.

its large circular dichroism, we also expect the frequency of maximum circular dichroism to be tuned by the pump. We connect the output of the waveguide to the second port of the VNA in order to measure the transmission response of the signal wave. The measured spectrum is shown in Fig. 5 for varying pump powers $\left(P_{p}\right)$ using $\omega_{p}=2 \pi \times 3.536 \mathrm{GHz}$. As expected, the circular dichroism resonance tunes monotonically with $P_{p}$, with a maximum tuning on the order of the resonance bandwidth. Therefore, we conclude that the main effect of the cross-phase modulation in our structure is in the frequency shift of the resonant chiral properties of the metamaterial. As a result, the dichroism and polarization rotation experienced by a wave of fixed frequency in the vicinity of the resonance can be modified using cross-phase modulation. This demonstrates that the concept of nonlinear optical activity, which was previously shown to be quite strong in metamaterials, ${ }^{19}$ is also applicable to multifrequency interaction processes.

\section{B. Four-wave mixing}

We next apply a pump (frequency $\omega_{p}$ ) and signal (frequency $\left.\omega_{s}\right)$ in order to generate a wave at the mix frequency $\omega_{n l}=$ $2 \omega_{p}-\omega_{s}$. By measuring in a circular basis, we seek to verify that Eqs. (7)-(9) are able to qualitatively predict the strength of the generated signal. We discretely sweep the fundamental frequencies across a range centered at the resonance frequency,
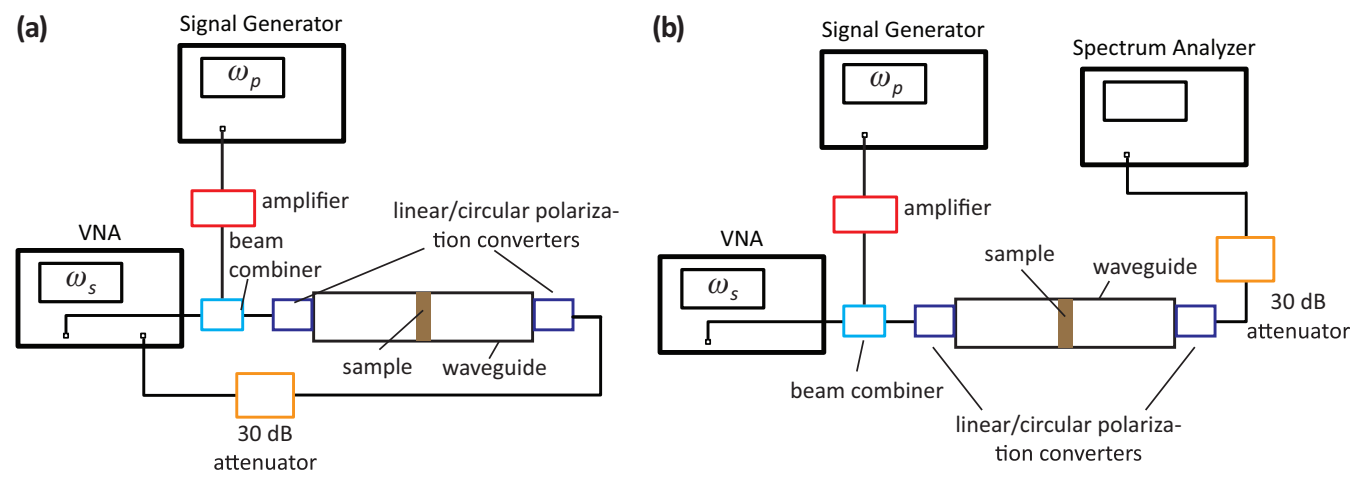

FIG. 4. (Color online) Schematics of the experimental setups used for studying (a) cross-phase modulation and (b) four-wave mixing in the varactor loaded metamaterials. 

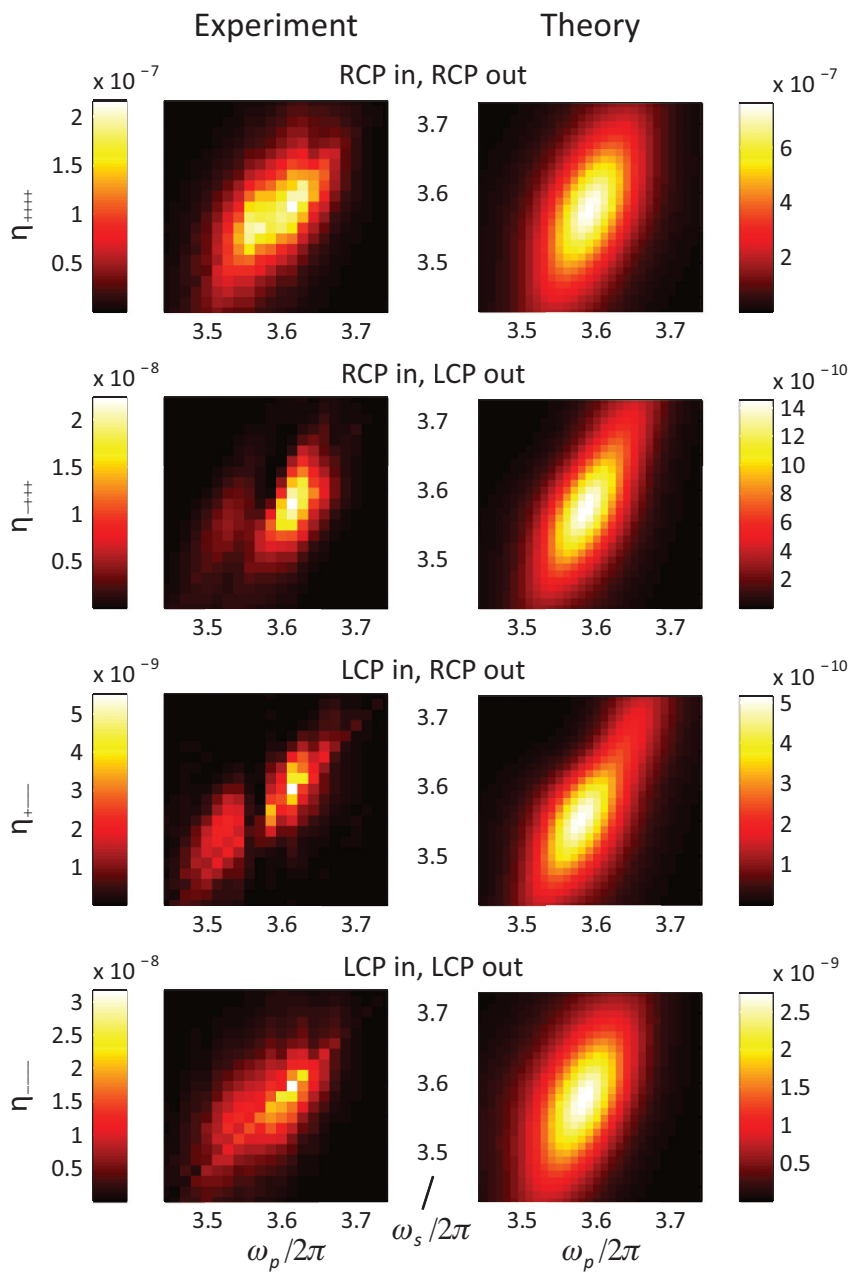

FIG. 6. (Color online) Coefficients of the four-wave mixing interactions $\eta\left(\mathrm{m}^{4} / \mathrm{W}^{2}\right)$ calculated from experiment (left) and simulation (right) for different combinations of circularly polarized waves.

measuring the power at $\omega_{n l}$ with a Rohde and Schwarz FSV30 spectrum analyzer. The pump and signal waves are both at a level of $15 \mathrm{~dB} \mathrm{~m}$ before entering the combiner. This power level is chosen to ensure that cross-phase modulation effects are weak. The measurement is repeated for all combinations of circularly polarized adapters and is plotted in Fig. 6 in terms of the efficiency, $\eta=P_{n l} A^{2} /\left(P_{p}^{2} P_{s}\right)$, where $A$ is the cross-sectional area of the waveguide. For comparison, we also plot $\eta$ calculated from simulations on a semi-infinite metamaterial slab, using the small-voltage expansion of the varactor's capacitance to model the mixing process within the nondepleted pump limit. ${ }^{32}$

The qualitative features and frequency dependence of the parametric four-wave mixing process show good agreement between theory and experiment. We can see from the experiments that the conversion of the RCP waves into RCP waves (the first row in Fig. 6) in our structure is an order of magnitude stronger than conversion of the LCP into LCP waves (the fourth row), which is of the same order as cross-polarization conversion of the RCP into LCP waves (the second row). The conversion coefficient for the LCP into RCP waves is another order of magnitude weaker. In general, such behavior is understandable from the linear properties of the structure. Indeed, the RCP waves excite resonances in the structure, and larger electric currents in the elements produce stronger nonlinear response in the form of four-wave mixing. The LCP waves are only weakly coupled to the structure's resonance, and therefore the nonlinear conversion coefficients are much weaker.

The discrepancies between the theory and experiment in the efficiency of the mixed polarization processes, $\eta_{-+++}$and $\eta_{+---}$, can be traced to linear polarization conversion in the metamaterial itself, demonstrated earlier to be on the order of $-10 \mathrm{~dB}$. However, the inequalities Eqs. (7)-(9) derived from the coupled-mode theory are still satisfied, since the mixing products involving pump and generated waves of different polarizations are greatly suppressed compared to those involving the same polarization. In addition, excitation of the structure by RCP waves clearly results in much greater efficiency, consistent with the transmission results in Fig. 3(b) showing much stronger interaction for this polarization. Further differences between theory and experiment can be attributed to dispersion in the waveguide used in the experiments, which is absent in the theory and reduces the circular dichroism exhibited by the sample.

\section{CONCLUSIONS}

We have studied the parametric interaction of pump and signal waves in a type of varactor-loaded nonlinear chiral metamaterial, including four-wave mixing and cross-phase modulation effects. We have elucidated several circular polarization selection rules based on a previously reported effective medium theory, subsequently verifying them through experiment and finite element simulations. Ultimately, we believe our results serve to both explore the vast parameter space available to nonlinear metamaterials as well as validate the previously developed nonlinear effective medium theory. We note that the presented structure can be extended to multiple layers in order to produce even greater chirality and nonlinear conversion or scaled to higher and more interesting frequencies by replacing the varactor diode with appropriate nonlinear crystals or substrates. ${ }^{9}$

\section{ACKNOWLEDGMENT}

This work was supported by the Australian Research Council.
${ }^{1}$ J. B. Pendry, Science 306, 1353 (2004).

${ }^{2}$ M. Kuwata-Gonokami, N. Saito, Y. Ino, M. Kauranen, K. Jefimovs, T. Vallius, J. Turunen, and Y. Svirko, Phys. Rev. Lett. 95, 227401 (2005).
${ }^{3}$ A. V. Rogacheva, V. A. Fedotov, A. S. Schwanecke, and N. I. Zheludev, Phys. Rev. Lett. 97, 177401 (2006).

${ }^{4}$ E. Plum, V. A. Fedotov, A. S. Schwanecke, N. I. Zheludev, and Y. Chen, Appl. Phys. Lett. 90, 223113 (2007). 
${ }^{5}$ M. Decker, M. W. Klein, M. Wegener, and S. Linden, Opt. Lett. 32, 856 (2007).

${ }^{6}$ T. Q. Li, H. Liu, T. Li, S. M. Wang, F. M. Wang, R. X. Wu, P. Chen, S. N. Zhu, and X. Zhang, Appl. Phys. Lett. 92, 131111 (2008).

${ }^{7}$ J. B. Pendry, A. J. Holden, D. J. Robbins, and W. J. Stewart, IEEE Trans. Microwave Theory 47, 2075 (1999).

${ }^{8}$ A. A. Zharov, I. V. Shadrivov, and Y. S. Kivshar, Phys. Rev. Lett. 91, 037401 (2003).

${ }^{9}$ N. I. Zheludev and Y. S. Kivshar, Nat. Mater. 11, 917 (2012).

${ }^{10}$ O. Hess, J. B. Pendry, S. A. Maier, R. F. Oulton, J. M. Hamm, and K. L. Tsakmakidis, Nat. Mater. 11, 573 (2012).

${ }^{11}$ I. V. Shadrivov, A. A. Zharov, and Y. S. Kivshar, J. Opt. Soc. Am. B 23, 529 (2006).

${ }^{12}$ D. Huang, A. Rose, E. Poutrina, S. Larouche, and D. R. Smith, Appl. Phys. Lett. 98, 204102 (2011).

${ }^{13}$ M. Lapine, M. Gorkunov, and K. H. Ringhofer, Phys. Rev. E 67, 065601 (2003).

${ }^{14}$ B. Wang, J. Zhou, T. Koschny, and C. M. Soukoulis, Opt. Express 16, 16058 (2008).

${ }^{15}$ A. Rose, D. Huang, and D. R. Smith, Phys. Rev. Lett. 107, 063902 (2011).

${ }^{16}$ T. Verbiest, S. Van Elshocht, M. Kauranen, L. Hellemans, J. Snauwaert, C. Nuckolls, T. J. Katz, and A. Persoons, Science 282, 913 (1998).

${ }^{17}$ V. Ostroverkhov, O. Ostroverkhova, R. G. Petschek, K. D. Singer, L. Sukhomlinova, and R. Twieg, IEEE J. Sel. Top. Quantum Electron. 7, 781 (2001).
${ }^{18}$ A. Persoons, Opt. Mater. Express 1, 5 (2011).

${ }^{19}$ I. V. Shadrivov, V. A. Fedotov, D. A. Powell, Y. S. Kivshar, and N. I. Zheludev, New J. Phys. 13, 033025 (2011).

${ }^{20}$ I. V. Shadrivov, Appl. Phys. Lett. 101, 041911 (2012).

${ }^{21}$ M. Ren, E. Plum, J. Xu, and N. I. Zheludev, Nature Commun. 3, 833 (2012).

${ }^{22}$ S. Larouche and D. R. Smith, Opt. Commun. 283, 1621 (2010).

${ }^{23}$ S. Larouche, A. Rose, E. Poutrina, D. Huang, and D. R. Smith, Appl. Phys. Lett. 97, 011109 (2010).

${ }^{24}$ A. Rose, S. Larouche, D. Huang, E. Poutrina, and D. R. Smith, Phys. Rev. E 82, 036608 (2010).

${ }^{25}$ A. Rose, S. Larouche, E. Poutrina, and D. R. Smith, Phys. Rev. A 86, 033816 (2012).

${ }^{26}$ A. Yariv, IEEE J. Quantum Electron. 9, 919 (1973).

${ }^{27}$ J. Liu, Photonic Devices (Cambridge University Press, New York, 2004).

${ }^{28}$ J. D. Joannopoulos, S. G. Johnson, J. N. Winn, and R. D. Meade, Photonic Crystals: Molding the Flow of Light, 2nd ed. (Princeton University Press, Princeton, 2008).

${ }^{29}$ S. Tretyakov, F. Mariotte, C. Simovski, T. Kharina, and J.-P. Heliot, IEEE Trans. Antennas Propag. 44, 1006 (1996).

${ }^{30}$ S. Tretyakov, A. Sihvola, and L. Jylh, Photonics and Nanostructures 3, 107 (2005).

${ }^{31}$ D.-H. Kwon, D. H. Werner, A. V. Kildishev, and V. M. Shalaev, Opt. Express 16, 11822 (2008).

${ }^{32}$ E. Poutrina, D. Huang, and D. R. Smith, New J. Phys. 12, 093010 (2010). 